\title{
Digitale Soziale Sicherung: Potenzial für die Plattformarbeit
}

\author{
Die soziale Absicherung in der Plattformarbeit weist gravierende Lücken auf. Das Konzept „Digitale \\ Soziale Sicherung“ zeigt, wie die Sozialversicherung auch in einen sehr kurzfristig agierenden, \\ (teilweise) internationalen Markt integriert werden kann. Dabei wird in die Plattformen ein digitaler \\ Mechanismus implementiert, der mit jeder Beendigung eines Jobs automatisch einen bestimmten \\ Prozentsatz des vereinbarten Entgelts als Sozialbeitrag abführt. Indem dieser Mechanismus an \\ der Plattform ansetzt, wird ein effektives digitales Quellenabzugsverfahren für Plattformarbeit \\ ermöglicht. Das System wäre offen für die Beteiligung (möglichst vieler) verschiedener Staaten. Die \\ nationale Sozialversicherung behält aber ihre Souveränität und Flexibilität.
}

Die Digitalisierung krempelt die Arbeitswelt in vielfacher Hinsicht um. Jobs fallen weg, andere entstehen neu, Berufe verändern sich, die Arbeitsbedingungen und die Arbeitsinhalte wandeln sich. Darüber hinaus geht es aber auch um die Art und Weise, wie Arbeitsmärkte funktionieren: Onlineplattformen organisieren Arbeitsmärkte digital, Angebot an und Nachfrage nach einzelnen Tätigkeiten werden zusammengebracht. Letztere sind extrem vielfältig, das Spektrum reicht etwa von Fahrdienstleistungen bis zu Programmierungen.

Plattformarbeit: viele Tätigkeiten, wenig soziale Absicherung

Die Breite von möglichen Tätigkeiten legt Wachstumspotenzial nahe. In der Tat zeigen Studien wie etwa die von Pesole et al. eine Beteiligung an Plattformarbeit, die bereits über diejenige in der Zeitarbeit hinausgeht. ${ }^{1}$ Aus Abbildung 1 ist für 15 EU-Staaten etwa ein Anteil von gut $2 \%$ der Bevölkerung im Erwerbsalter zu entnehmen, die Plattformarbeit als Hauptjob betreibt. Der Kreis derjenigen, die zumindest in relevantem Umfang über Plattformen tätig sind, ist noch deutlich größer. Aus Arbeitsmarktperspektive könnte Plattformarbeit durchaus Potenzial haben. Plattformen können etwa die Markttransparenz erhöhen, Transaktionskosten senken und die Flexibilität erhöhen. Sie könnten prinzipiell auch Möglichkeiten des unabhängigen

(c) Der/die Autor(en) 2020. Open Access: Dieser Artikel wird unter der Creative Commons Namensnennung 4.0 International Lizenz (http:// creativecommons.org/licenses/by/4.0/deed.de) veröffentlicht.

Open-Access wird durch die ZBW - Leibniz-Informationszentrum Wirtschaft gefördert.

1 A. Pesole, M. C. Urzí Brancati, E. Fernández-Macías, F. Biagi, I. González Vázquez: Platform Workers in Europe - Evidence from the COLLEEM Survey. EUR - Scientific and Technical Research Reports, Publications Office of the European Union, 2018. und selbstbestimmten Arbeitens bieten. Dem stehen aber auch Nachteile gegenüber. So zeigen mittlerweile etliche Studien, dass die soziale Absicherung der Betroffenen gravierende Lücken aufweist. Selbst wenn die Absicherung der Personen in einem Land rechtlich gesehen möglich ist, fällt die effektive Abdeckung schwach aus.

\section{Das Konzept der Digitalen Sozialen Sicherung}

Die Herausforderung liegt darin, dass man es mit einem teils international integrierten, extrem flexiblen Markt mit einer Vielzahl von Kleinstjobs und ständig wechselnden Vertragspartnern zu tun hat. Die digitale Plattformökonomie stellt die soziale Sicherung also vor Probleme. Das Konzept der „Digitalen Sozialen Sicherung“ (DSS-Konzept) zeigt aber, wie man sich die neue digitale Marktform zunutze machen kann, um Sozialversicherungen auch in einem solchen Umfeld zu organisieren. ${ }^{2}$

Das DSS-Konzept orientiert sich am Begriff des zentralen Marktakteurs. Zahlreiche heutige Sozialversicherungssysteme sind im Zuge der industriellen Transformation entstanden. Diese führte zu einer Organisation vieler Beschäf-

2 E. Weber: Setting out for Digital Social Security, ILO Research Department, Working paper, Nr. 34, 2018; ders.: Ein Konzept für Digitale Soziale Sicherung in der Plattformarbeit, in: Deutsche Rentenversicherung, 74. Jg. (2019), H. 3, S. 247-258.

Prof. Dr. Enzo Weber ist Forschungsbereichsleiter am Institut für Arbeitsmarkt- und Berufsforschung (IAB) und lehrt an der Universität Regensburg. 


\section{Abbildung 1}

\section{Umfang der Plattformarbeit in ausgewählten EU-Ländern}

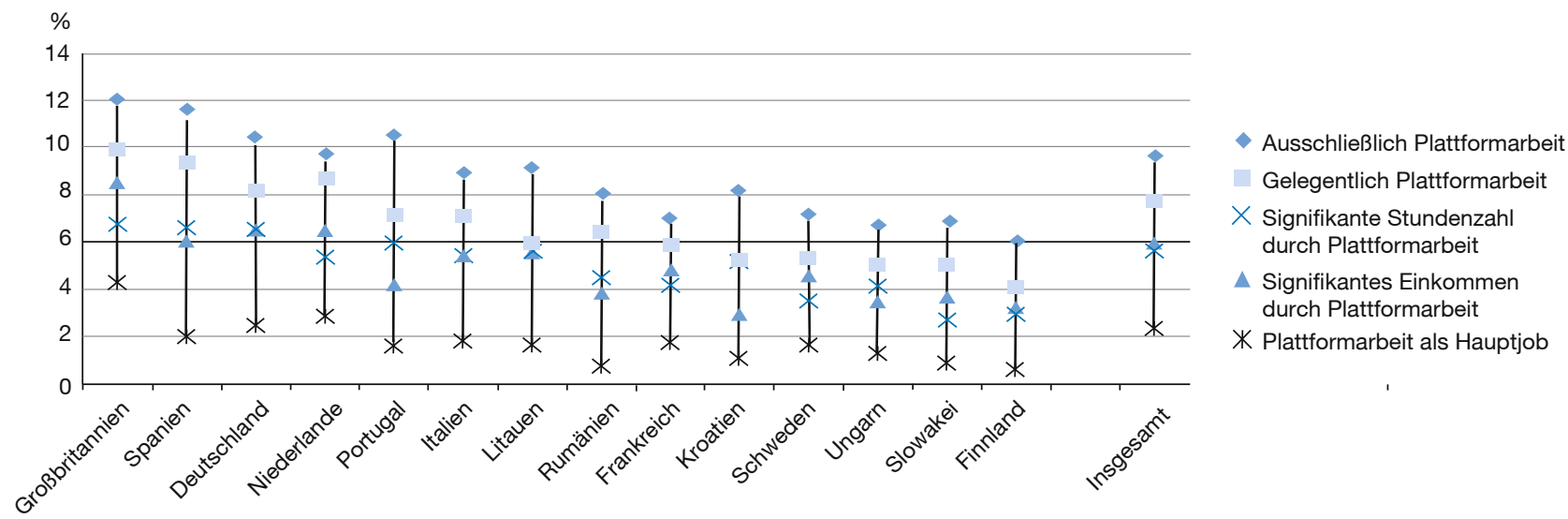

Quelle: A. Pesole, M. C. Urzí Brancati, E. Fernández-Macías, F. Biagi, I. González Vázquez: Platform Workers in Europe - Evidence from the COLLEEM Survey. EUR - Scientific and Technical Research Reports, Publications Office of the European Union, 2018.

tigter in Firmen. Genau dies machte man sich zunutze, indem Schutzstandards und Sozialversicherungen direkt bei den Firmen angesetzt wurden. Auch heute gibt es wieder eine Transformation, diesmal durch die Digitalisierung. Entsprechend sollte man nun direkt bei den neuen zentraIen Akteuren der Online-Wirtschaft ansetzen, nämlich den Plattformen.

Hier liegen die relevanten Informationen vor, über Auftragnehmer, Auftraggeber und das vereinbarte Entgelt. Letzteres muss üblicherweise vorab deponiert werden, und Plattformen verlangen teils erhebliche Gebühren. Sie wären also auch in der Lage, die Abführung von Sozialversicherungsbeiträgen durchzusetzen.

Dementsprechend sieht das DSS-Konzept vor, direkt in die Plattformen einen digitalen Mechanismus zu implementieren, der mit jeder Beendigung eines Jobs einen bestimmten Prozentsatz des vereinbarten Entgelts auf das DSSKonto des Plattformarbeiters überweist (vgl. Abbildung 2). Dies ist Teil eines digitalen Systems persönlicher Konten, auf denen die Beiträge aus allen Plattformaufträgen kumuliert werden. Dieses würde eine gemeinsame Infrastruktur bereitstellen und könnte von einer bereits bestehenden internationalen Institution wie der Internationale Arbeitsorganisation (ILO) oder der Weltbank - oder auch EU-intern - verwaltet werden. Der Beitrag könnte beispielsweise zur Hälfte als Zahlung zusätzlich zur vereinbarten Vergütung und zur Hälfte als Abzug von der vereinbarten Vergütung finanziert werden.

In regelmäßigen Abständen werden die gesammelten Beiträge dann in das Sozialversicherungssystem des Wohnsitzlandes des Plattformarbeiters transferiert. Hier werden
Ansprüche in etablierten Strukturen generiert. Die nationale Sozialversicherung behält also ihre Souveränität und Flexibilität. Es wäre keinerlei Harmonisierung oder Standardisierung sozialversicherungspolitischer Regelungen notwendig.

So würde die Verteilung der Beiträge auf verschiedene Sozialversicherungszweige weiterhin länderspezifisch erfolgen. Zentral wären sicherlich die großen Zweige Renten- und Krankenversicherung. Gerade für den Aufbau von Rentenansprüchen könnten auch nebenberufliche Tätigkeiten in der Plattformarbeit einen Beitrag leisten, wie auch Plattformarbeit etwa neben dem Studium. Dies folgt dem Prinzip, die soziale Sicherung an die Erzielung von Einkommen zu knüpfen und dafür auch verschiedene Quellen zu berücksichtigen. ${ }^{3}$ Auch Arbeitslosigkeit ist ein für die Plattformarbeit durchaus relevantes Phänomen. So zeigen Forschungsergebnisse, dass keineswegs von einem stets verfügbaren unerschöpflichen Pool von Aufträgen auszugehen, sondern Arbeit häufig auch unzureichend vorhanden ist. Dementsprechend wäre auch eine Einbeziehung in die Arbeitslosenversicherung in Betracht zu ziehen - umso mehr, wenn diese auch Weiterbildungsleistungen umfasst, zu denen Plattformarbeiter ohne betrieblichen Kontext keinen organisierten Zugang haben. Die Transparenz der DSS-Beitragszahlungen würde das Problem lösen, Arbeitslosigkeit für Freelancer festzustellen. Schließlich ist auch das Risiko von Arbeitsunfällen zu bedenken. Als Empfänger kämen prinzipiell auch private Versicherungen bzw. Nicht-Regierungs-Institutionen infrage.

3 Vgl. etwa P. Schoukens, A. Barrio: The changing concept of work: When does typical work become atypical?, in: European Labour Law Journal, 8. Jg. (2017), H. 4, S. 306-332. 
Abbildung 2

Digitale Soziale Sicherung

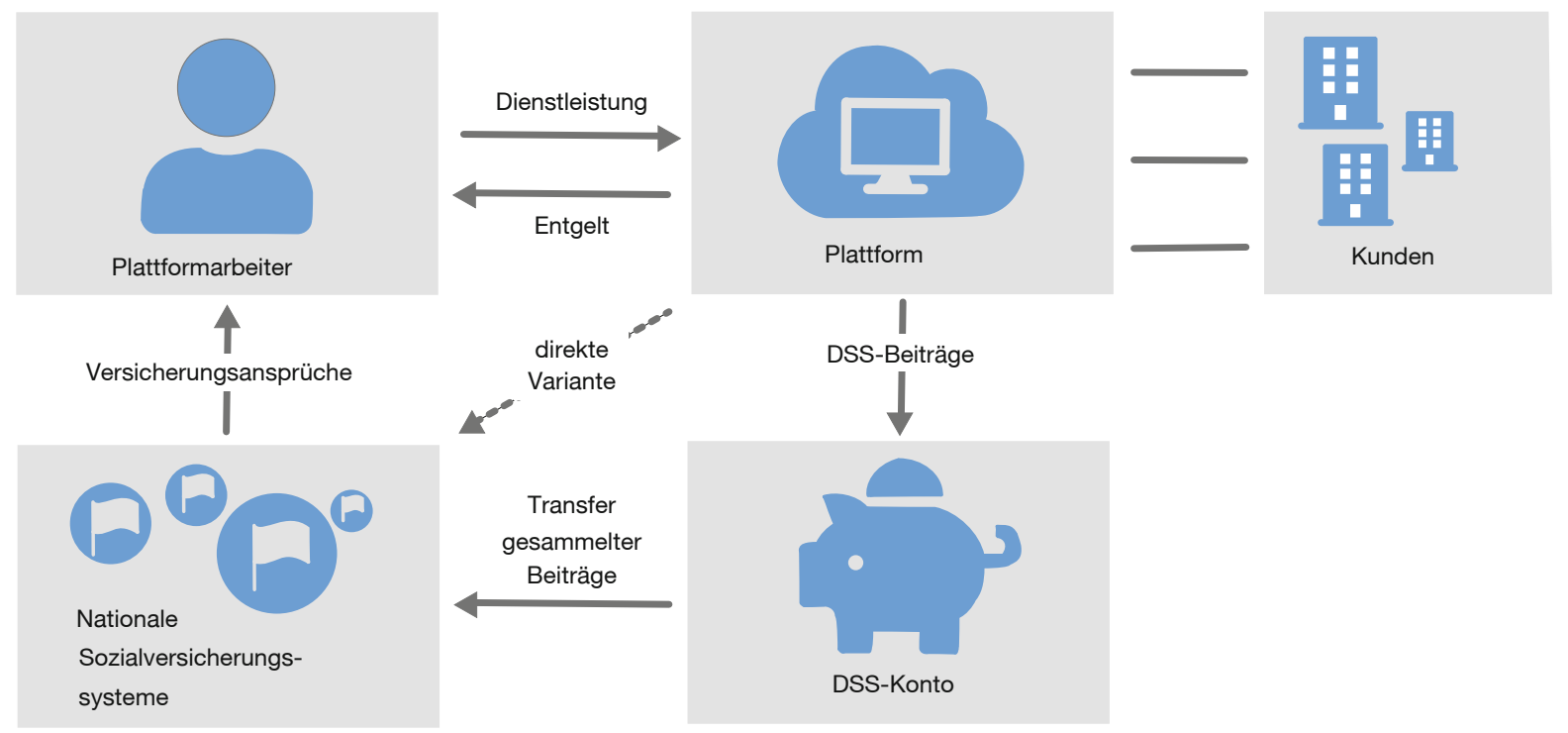

Quelle: eigene Darstellung; Icons entnommen von https://fontawesome.com/ (25.2.2020), Lizenz CC BY 4.0.

Als einfachste und transparenteste Lösung käme ein einheitlicher DSS-Beitragssatz in Betracht. Dieser würde naturgemäß nicht die Vielfalt der nationalen Sätze abbilden. Dem könnte Rechnung getragen werden, indem die individuellen Leistungsansprüche entsprechend skaliert würden. Läge der DSS-Beitrag z.B. $5 \%$ unter demjenigen im jeweiligen nationalen System, so wären auch die daraus resultierenden Leistungsansprüche (außer in der Krankenversicherung) um $5 \%$ niedriger. Eine solche Skalierung wird beispielsweise in der freiwilligen gesetzlichen Renten- und Unfallversicherung für Selbständige in Deutschland bereits heute praktiziert. Wenn länderspezifische Beitragssätze präferiert werden, wäre auch das organisierbar - unter Umständen beschränkt auf ortsgebundene Tätigkeiten.

\section{Ein digitales Quellenabzugsverfahren - und Varianten}

Das DSS-Konzept bietet die Möglichkeit, ein digitales Quellenabzugsverfahren für die Plattformwirtschaft zu implementieren. Dies stellt eine soziale Absicherung automatisch sicher, was gerade vor dem Hintergrund einer meist starken Cash-Präferenz wie auch typischer Informationsdefizite einen wesentlichen Vorteil darstellt. Überraschende Nachforderungen und andere Unsicherheiten werden durch das DSS-Konzept vermieden, ebenso wie Beitragshinterziehung. Das Konzept bietet ein hohes Maß an Effizienz für Plattformarbeiter und Kunden, aber aufgrund seiner digitalen Ausgestaltung ebenso für Plattformen als Digitalunternehmen. Es sind genau diese Gründe, aus denen viele Länder für die abhängige Beschäftigung ein Quellenabzugsverfahren verwenden.

Die Beteiligung wäre verpflichtend zu gestalten. Durch die Möglichkeit, Aktivitäten von nicht-kooperierenden Plattformen in ihrem Hoheitsgebiet zu beschränken, hätten Staaten generell eine Handhabe zur Durchsetzung. Da Plattformen als Geschäftsmodell auf Offenheit und Netzwerkeffekte angewiesen sind, wäre selbst eine bloße Illegalisierung schon ein erhebliches Druckmittel. Die Regelbefolgung ließe sich etwa durch Inkognito-Stichproben und Steuerinformationen überprüfen. Die organisatorische Durchschlagskraft und Verhandlungsmacht würde mit der Zahl der teilnehmenden Länder zunehmen. Hierbei könnte auch die Europäische Union eine produktive Rolle spielen.

Möglich wären auch Varianten des Grundmodells. So könnten die Beiträge von den Plattformen ohne Zwischenschaltung eines DSS-Kontensystems direkt an die nationalen Systeme fließen (vgl. „direkte Variante“ in Abbildung 2), so wie es zunehmend etwa bei der Abführung von Umsatzsteuern geschieht. Die Beitragskonten würden dann komplett in nationaler Regie geführt. In diesem Fall sollten technische Standards für Schnittstellen und Prozesse definiert werden, wie sie im DSS-Kontensystem ohnehin zum Einsatz kämen. Denkbar wäre, dass die einzelnen Nationalstaaten entweder das DSS-Kontensystem in Anspruch zu nehmen oder für die direkte Variante optieren. Grundsätzlich möglich wäre auch, die Beiträge nicht von Job zu Job sondern traditionell kumuliert am Monatsende abzuführen. 
Eine weitere Variante könnte vorsehen, dass keine Sozialversicherungsbeiträge direkt von den Plattformen transferiert, sondern lediglich Informationen über erzielte Einkommen der Personen übermittelt werden. Die Zahlungsverpflichtung läge dann beim Individuum. Über einen automatisierten Bankeinzug ließe sich der Prozess vereinfachen; eine solche Variante wird etwa gerade in Frankreich erprobt. Während auch dies ein gangbarer Weg wäre, kämen einige wesentliche Vorteile des Quellenabzugsverfahrens dabei nicht zum Tragen. Insbesondere würden Unsicherheiten zu Zahlungsverpflichtungen und das Entstehen von Beitragsschulden speziell bei Illiquidität nicht vermieden. Die Internalisierung der Sozialversicherungskosten bei der Lohnfindung, die beim Quellenabzugsverfahren sehr direkt erfolgt, würde zulasten der Plattformarbeiter erschwert. Beiträge vonseiten der Plattform bzw. der Kunden würden nicht erfolgen.

Weiterhin könnte man daran denken, die soziale Sicherung an die Besteuerung zu knüpfen. So findet eine Einkommensermittlung bereits im Rahmen der Steuererklärung statt. Zu bedenken ist dabei der vermutlich erhebliche Anteil von Schwarzarbeit bei Plattformtätigkeiten. Zudem weisen Einkommensteuererklärungen oft große Verzögerungen auf. Die Probleme eines fehlenden Quellenabzugsverfahrens kämen auch hier zum Tragen. Sollte ein solches steuerlich organisiert werden, ließe sich aber ein gemeinsames Vorgehen mit der Sozialversicherung vorstellen. Die Steuererklärung könnte zudem für mögliche Beitragsrückzahlungen genutzt werden, etwa im Falle besonders hoher Werbungs- bzw. Investitionskosten.

Möglicherweise sind in verschiedenen bestehenden Sozialsystemen eine obligatorische Beteiligung von Plattformen bzw. eine Einbeziehung entsprechender Einkünfte in die soziale Sicherung rechtlich nicht unmittelbar realisierbar. In solchen Fällen könnte das Konzept in einer ersten Phase auch vorsehen, dass der Plattformarbeiter die Plattform autorisiert an dem DSS-Konzept teilzunehmen - also Beiträge abzuführen bzw. Informationen zu übermitteln. Eine umfassende soziale Absicherung und Risikoverteilung lässt sich perspektivisch aber nur mit einer verpflichtenden Sozialversicherung erreichen.

\section{Soziale Sicherung für die Zukunft der Plattformarbeit}

Der arbeitsrechtliche Status von Plattformarbeitern unterliegt erheblichen Unsicherheiten. Das DSS-Konzept ist jedoch in jedem Falle sinnvoll: Für diejenigen, die als abhängig beschäftigt klassifiziert werden, bietet es ein effizientes Instrument, um soziale Sicherung in einem amorphen Arbeitsmarkt zu organisieren. Und für Selbständige und den großen Graubereich füllt das Konzept eine tatsächliche Lücke: Die effektive Abdeckung der sozialen Sicherung könnte entscheidend erhöht werden. So würde sich DSS auch eignen, die in Deutschland geplante Neuregelung der Altersvorsorge für Selbständige effizient umzusetzen.

Plattformarbeit wird oft sehr niedrig entlohnt, was die Finanzierung der sozialen Sicherung erschwert. Ohne eine soziale Sicherung werden prekäre Situationen aber beim Eintritt von Notlagen noch verschärft, individuelle berufliche Zukunftsinvestitionen erschwert und die Löhne in einem unregulierten Markt auf ein nicht nachhaltiges Niveau gedrückt. Auch bestehen Fehlanreize, Jobs auf Plattformen zu verlagern, um über Scheinselbständigkeit Sozialversicherungskosten zu sparen. Das DSS-Konzept kann in dieser Hinsicht eine positive soziale Entwicklung initieren und dazu beitragen, die Potenziale von Plattformarbeit auf einer nachhaltigen Basis zu entwickeln.

Die Implementierung des DSS-Konzepts könnte über Experimente beginnen, die es Marktteilnehmern und politischen Entscheidungsträgern ermöglichen, Erfahrungen mit dem neuen Instrument zu sammeln und Akzeptanz zu generieren. Sobald institutionelle Strukturen geschaffen und weiterentwickelt wurden, könnte der Anwendungsbereich erweitert werden. Für die vollständige Implementierung sind „First Mover" erforderlich, die ein möglichst großes Gewicht in die Waagschale werfen und Standards setzen können. Dabei ist es zu empfehlen, das System im Grundsatz für weitere Teilnehmer-Länder offen auszulegen. Dies könnte über die Zeit in eine multilaterale Regelung ähnlich dem internationalen Seearbeitsübereinkommen der ILO hineinwachsen. Politisch ist die Organisation der sozialen Sicherung Sache des nationalen Gesetzgebers - aber die neue Herausforderung der Plattformarbeit ist für alle gleich.

Title: Digital Social Security: Potential for Platform Work

Abstract: Social security in platform work has serious gaps. 'Digital Social Security' shows how social insurance can also be organised in a market with a very short-term and (partially) internationally integrated character. A digital mechanism is implemented in the platforms, which automatically pays a certain percentage of the agreed remuneration as a social contribution each time a job is finished. By integrating the DSS mechanism directly into the platform, an effective pay-as-you-go procedure for platform work is made possible. The system would be open to the participation of as many different states as possible. However, the national social security system remains fully sovereign and flexible.

JEL Classification: J21, J28, 039 